\title{
ESTÁDIOS EVOLUTIVOS DE TRIPANOSSOMAS DE HIPOSTOMUS PUNCTATUS VALENCIENNES (OSTEICHTHYES, LORICARIIDAE) EM INFECÇÃO NATURAL DE BATRACOBDELLA GEMMATA BLANCHARD (HIRUDINEA, GLOSSIPHONIIDAE)
}

\author{
Marta D'Agosto ${ }^{1}$ \\ Nicolau Maués da Serra-Freire ${ }^{2}$
}

\begin{abstract}
EVOLUTIVE STAGES OF TRIPANOSOMES OF HIPOSTOMUS PUNCTATUS VALENCIENNES (OSTEICHTHYES, LORICARIIDAE) IN NATURAL INFECTION OF BATRACOBDELLA GEMMATA BLANCHARD (HIRUDINEA, GLOSSIPHONIIDAE). Seventeen leeches obtained from armoured catfish (Hypostomus punctatus Valenciennes, 1840) infected with Trypanosoma spp. were examined. It was observed the presence of tripomastigotes, epimastigotes and amastigotes forms as well as dividing forms in the proboscis and in the stomach, different from the ones found in the vertebrate host as regards the morfometric features and developing forms. The examination of the contents of rectum did not show tripanosomes. These facts seem to demonstrate that Batracobdella gemmata (Blanchard, 1900) is an invertebrate host of trypanosomes and the transmission is done by means of inoculation.

KEY WORDS. Osteichthyes, Loricariidae, Hirudinea, Glossiphoniidae, Tripanosomes
\end{abstract}

Formas de desenvolvimento de tripanosomas de peixes em sanguessugas foram descritas por diversos autores, como BRUMPT (1904), que ao examinar conteúdo do trato digestivo de Hemiclepsis marginata, assinalou que as formas observadas eram de reduzidas dimensões e morfologicamente distintas das encontradas no hospedeiro vertebrado. Em outros estudos, BRUMPT (1905, 1906a) atribuiu importância taxonômica ao conhecimento dos vetores e modo de evolução do parasito em sanguessugas, descrevendo novas espécies com base neste critério (BRUMPT, 1906b). ROBERTSON (1910) propôs que as formas de evolução de Trypanosoma rajae em Pontobdella muricata estariam relacionadas ao estágio de digestão na sanguessuga. Este mesmo autor em 1912, observou a multiplicação de Trypanosoma granulosum em $H$. marginata, fazendo comentários sobre o período após o qual a infecção se transmitiria biologicamente.

$\mathrm{Na}$ tentativa de identificar o vetor de tripanosoma de peixes na África, trabalhando com sanguessugas BAKER (1960) e ABOLORIN (1970) concluíram que espécies do gênero Batracobdella poderiam ser os vetores.

1) Departamento de Zoologia, Universidade Federal de Juiz de Fora, 36036-300 Juiz de Fora, Minas Gerais, Brasil.

2) Departamento de Parasitologia Animal, Universidade Federal Rural do Rio de Janeiro, 23851-970 Itaguaí, Rio de Janeiro, Brasil. 


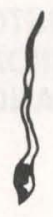

d

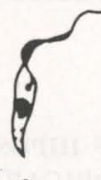

e

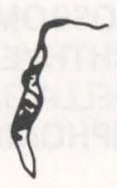

b
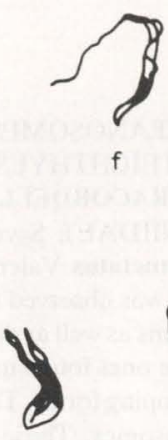

1

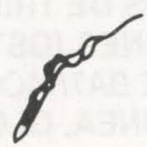

c
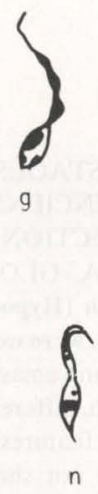

$n$
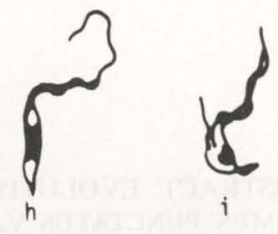

m

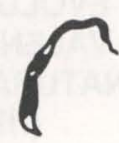

d

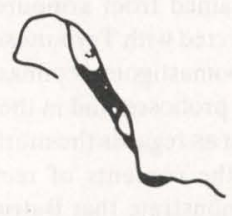

0
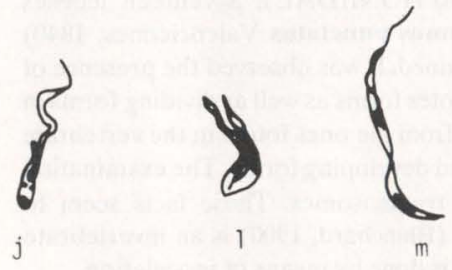

$10 \mu$

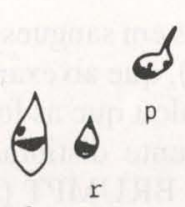

q

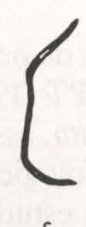

S

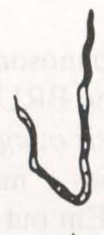

t
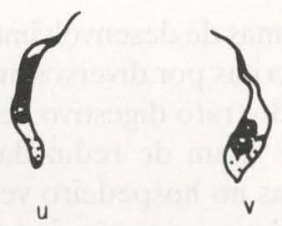

Fig. 1. Tripanosomas do estômago de sanguessugas Batracobdella gemmata provenientes do Lago Açú, Universidade Federal Rural do Rio de Janeiro. (a-d) Tripomastigotas; (e-f) epimastigotas; (g-p) formas da divisão; (p-r) amastigotas; (s-t) formas alongadas; (u-v) formas alteradas.

O ciclo evolutivo de Trypanosoma murmanensis e seu desenvolvimento em sanguessugas marinhas do gênero Johanssonia foi descrito por KHAN (1976), onde a infectividade desses tripanosomas às sanguessugas positivas foi de 24 horas a 93 dias após a infecção dos peixes (KHAN, 1977). O acompanhamento da infecção por tripanosoma em $H$. marginata foi realizado por QUADRI (1962a), NEEDHAM (1969) e por LECTH $(1977,1980)$, que estabeleceram o ciclo evolutivo e descreveram as divisões e as diferenças morfológicas e morfométricas de Trypanosoma danilewskyi, T. tincae e T. cobitis no estômago e probóscide da referida sanguessuga.

No Brasil BARA \& SERRA FREIRE (1984) observaram a presença de 
formas de desenvolvimento de tripanosomas no estômago e probóscide de sanguessugas do gênero Diplobdella, sendo esta a primeira referência de um vetor de tripanosomas de peixe no país. Em 1985, esses autores corrigiram a citação do gênero da sanguessuga comprovado por eles como hospedeiro intermediário de tripanosomas de peixes dulceaquícolas, informando que se tratava do gênero Batracobdella.

O presente trabalho teve como objetivos apresentar descrições morfológicas e morfométricas de formas de tripanosoma observadas em infecção natural de B. gemmata (Blanchard, 1900) recolhidas sobre $H$. punctatus.

\section{MATERIAL E MÉTODOS}

Sanguessugas da espécie $B$. gemmata removidas do hospedeiro $H$. punctatus pescados no Lago-Açú do Campus da Universidade Federal Rural do Rio de Janeiro, foram fixadas em concentrações crescentes de etanol até $10 \%$ e conservadas em etanol a $80 \%$; outras foram mantidas vivas em frascos aerizados para teste de transmissão de tripanosomas à peixes não infectados, e 17 destas dissecadas em uma tentativa de se localizar formas de tripanosoma em desenvolvimento no tubo digestivo. Esfregaços foram preparados com a probóscide e com o conteúdo do estômago e do reto, fixados em metanol e corados com Giemsa.

Para as medidas, tomadas em um, da largura corporal (L) e da distância do cinetoplasto à extremidade posterior $(\mathrm{CP})$ foi utilizada ocular micrométrica; comprimento total do corpo (CT), comprimento do corpo (CC), comprimento do flagelo livre $(\mathrm{F})$, distância do meio do núcleo à extremidade posterior $(\mathrm{PN})$ e distância do meio do cinetoplasto ao meio do núcleo (NC) foram medidos segundo DIAS \& FREITAS (1943), assim como foi calculado o índice nuclear (IN).

Para a representação gráfica utilizou-se o diagrama de Dice-Leraas (DICE \& LERAAS, 1936).

\section{RESULTADOS}

No exame dos esfregaços de conteúdo estomacal e da probóscide constatou-se a presença de tripanosomas em todas as sanguessugas dissecadas. Tanto no estômago como na probóscide foram observadas formas tripomastigotas, epimastigotas, amastigotas e grande número de formas em divisão. O exame do conteúdo do reto não demonstrou tripanosomas. As formas tripomastigotas no estômago de sanguessugas se caracterizam pelas reduzidas dimensões em relação às sanguíneas do peixe (Fig. 1a-d) e pelo flagelo livre, que pode estar presente ou não, com descolamento do cinetoplasto e do núcleo em sentido convergente, com diminuição da distância entre eles. O cinetoplasto distanciou-se da extremidade posterior, porém a localização do núcleo é claramente posterior, apresentando índice nuclear médio de 0,5 (Tab. I). A duplicação do cinetoplasto, embora possa ser observada com localização posterior ao núcleo 


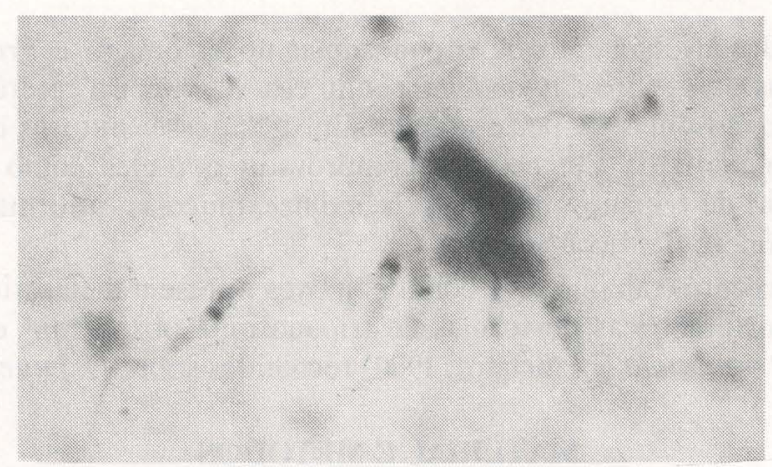

Fig. 2. Fotomicrografia de tripanosomas do estômago de Batracobdella gemmata. Aumento de $1600 x$.

Tabela I. Biometria (m) de tripanosomas no estômago e na probóscide de sanguessugas naturalmente infectadas, provenientes do Lago Açú da Universidade Federal Rural do Rio de Janeiro.

\begin{tabular}{lccccc}
\hline \multirow{2}{*}{ Caracteres } & \multicolumn{2}{c}{ Estômago } & & \multicolumn{2}{c}{ Probóscide } \\
\cline { 2 - 3 } \cline { 5 - 6 } & Tripomastigotas & Epimastigotas & & Tripomastigotas & Epimastigotas \\
\hline Comprimento do corpo & $14,3 \pm 2,3$ & $13,5 \pm 2,4$ & & $15,1 \pm 2,7$ & $12,1 \pm 3,4$ \\
& $(10,3-17,1)$ & $(10,8-17,7)$ & & $(11,4-19,4)$ & $(7,4-17,7)$ \\
Flagelo livre & $2,9 \pm 2,3$ & $3,5 \pm 2,6$ & & $2,6 \pm 2,9$ & $3,6 \pm 2,5$ \\
& $(0,0-6,8)$ & $(0,0-11,4)$ & & $(0,0-9,1)$ & $(1,1-8,6)$ \\
Comprimento total do & $18,4 \pm 5,6$ & $16,9 \pm 3,2$ & & $17,7 \pm 4,4$ & $15,7 \pm 3,8$ \\
corpo & $(10,8-21,7)$ & $(10,7-26,2)$ & & $(11,4-25,1)$ & $(10,3-23,4)$ \\
Largura do corpo & $1,6 \pm 0,6$ & $1,4 \pm 0,3$ & & $2,0 \pm 0,7$ & $1,7 \pm 0,5$ \\
& $(0,9-2,6)$ & $(1,0-2,2)$ & & $(1,0-3,1)$ & $(1,0-2,5)$ \\
Índice nuclear & $0,5 \pm 0,18$ & $0,4 \pm 0,17$ & & $0,4 \pm 0,22$ & $0,3 \pm 0,17$ \\
& $0,2-0,8)$ & $(0,0-0,7)$ & & $(0,1-0,8)$ & $(0,1-0,6)$ \\
Cinetoplasto àn extremidade & $2,3 \pm 1,5$ & $4,3 \pm 1,3$ & & $0,9 \pm 0,6$ & $4,3 \pm 1,8$ \\
posteriors & $(0,6-5,1)$ & $(1,1-6,8)$ & & $(0,4-2,3)$ & $(1,7-6,8)$ \\
\hline
\end{tabular}

(Fig. 1g) é mais característica em formas epimastigotas (Fig. 1h-i). Epimastigotas foram observadas em divisão ou não (Figs 1e-f, 2). Também foram comprovadas diferentes fases de divisão no estômago (Fig. 1g-p). Registrou-se a presença de formas amastigotas (Fig. 1p-r). Entre as alterações morfológicas destacam-se o alargamento da extremidade posterior e a concentração nessa região de granulações citoplasmáticas (Fig. 1u-v). Embora com pouca freqüência foram constatadas formas finas e longas $(28,5 \times 0,76 \mathrm{~mm})$ e com granulações (Fig. 1s-t).

Na probóscide encontrou-se variação quanto a morfologia e mensuração das formas provenientes de diferentes sanguessugas (Figs 3-5). Em algumas, predominaram as formas epimastigotas finas, com membrana ondulante pouco definida, assim como flagelo livre, quando presente (Fig. 3a-d), observando-se epimastigotas em divisão (Fig. 3i-j) e tripomastigotas (Fig. 3e-h). Em outras, 
houve predomínio de formas tripomastigotas (Fig. 4d-g), sendo também observadas formas epimastigotas (Fig. 4a-c) e em divisão (Figs 4h-i, 5b).

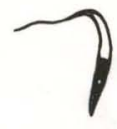

a

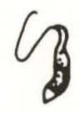

b

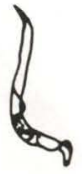

$f$

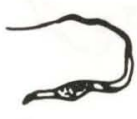

$\mathrm{e}$

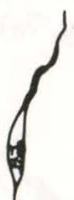

C

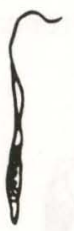

d

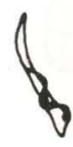

g

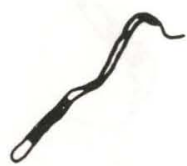

h

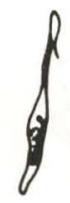

i

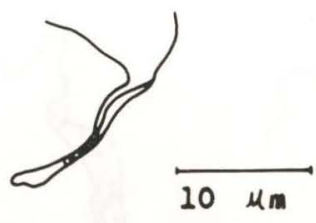

j

Fig. 3. Tripanosomas da probóscide de Batracobdella gemmata provenientes do Lago Açú, Universidade Federal Rural do Rio de Janeiro. (a-d) Epimastigotas; (e-h) tripomastigotas; (i-j) formas em divisão.

Nas sanguessugas em que se evidenciou, na probóscide, maior número de epimastigotas em relação às tripomastigotas, estas estavam em grande concentração (Fig. 5a), finas e longas, com flagelo livre, este sempre presente e bem desenvolvimento (Fig. 5c).

Os dados biométricos das formas tripomastigotas do estômago e da probóscide de sanguessugas estão apresentados na tabela I. As medidas de comprimento e de largura do corpo para tripomastigotas e epimastigotas do estômago e da probóscide, não tiveram diferenças significativas (Fig. 6a-b). 
A comparação do comprimento do corpo de tripomastigotas da circulação de peixe, do estômago e da probóscide de sanguessugas demonstrou redução significativa no hospedeiro invertebrado (Fig. $7 \mathrm{~b}$ ); quanto a largura, não houve diferença significativa (Fig. 7a).

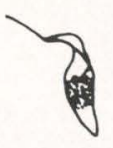

a

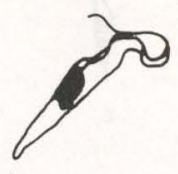

b

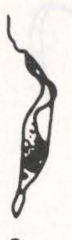

C

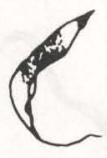

d

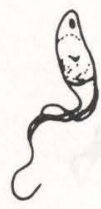

e
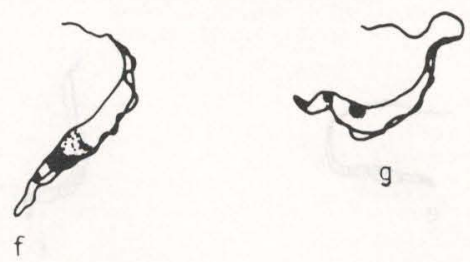

g

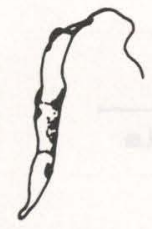

h

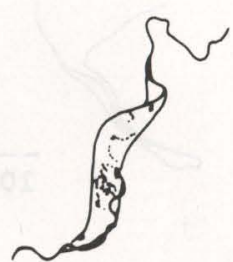

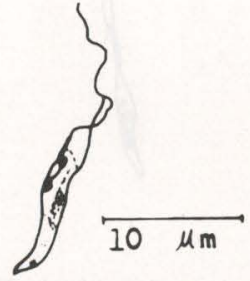

j

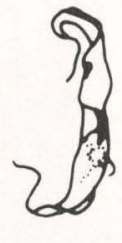

1

Fig. 4. Tripanosomas da probóscide de Batracobdella gemmata provenientes do Lago Açú, Universidade Federal Rural do Rio de Janeiro. (a-c) Epimastigotas; (d-g) tripomastigotas; (h-l) formas em divisão.

\section{DISCUSSÃO}

A redução de tamanho do flagelado ao passar do hospedeiro vertebrado para o invertebrado já havia sido destacada por QUADRI (1962a) e por LETCH (1980) ao acompanharem a evolução de tripanosomas de peixes em $H$. marginata. Resultados semelhantes também foram registrados quanto ao pro- 


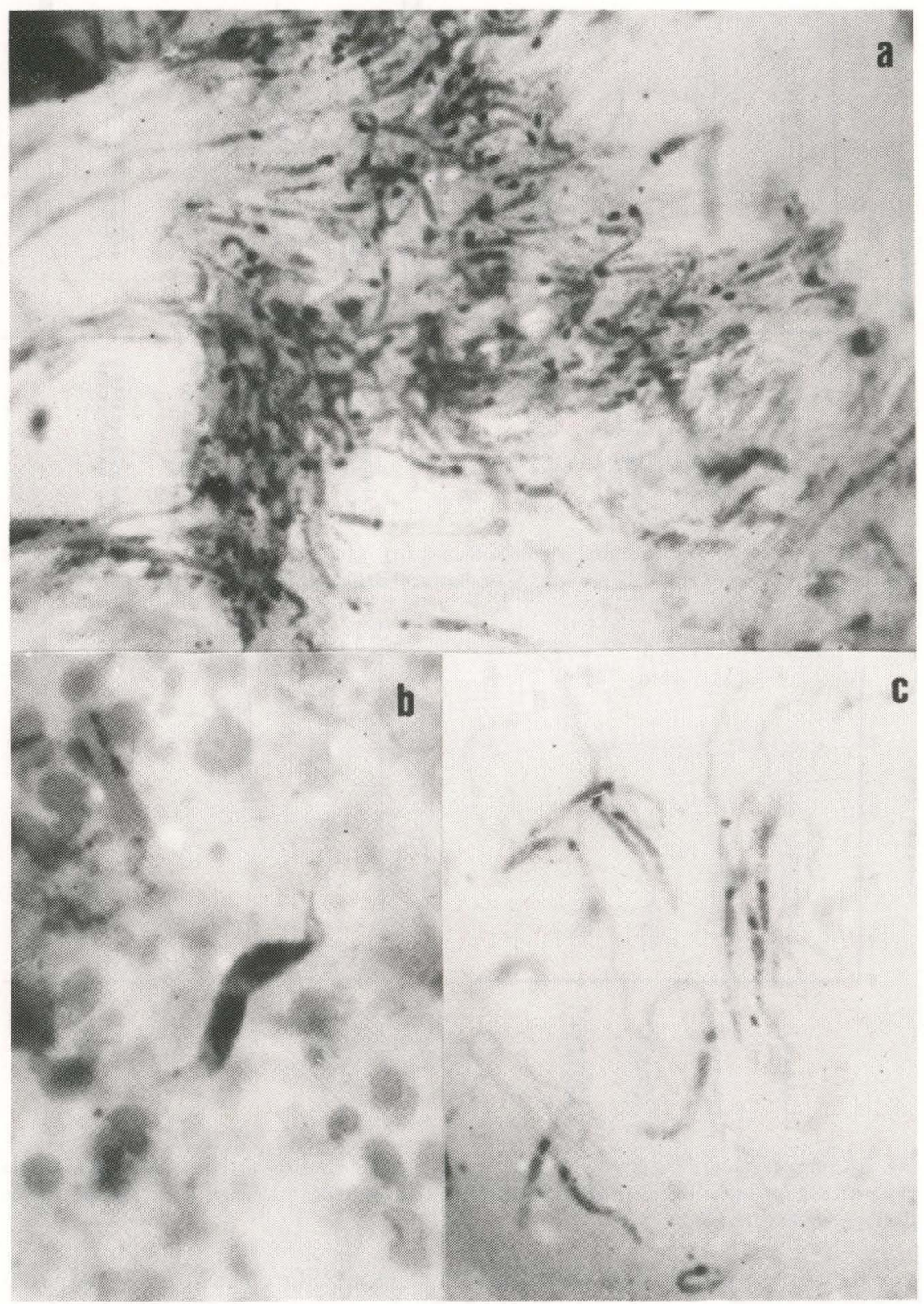

Fig. 5. Fotomicrografias de tripanosomas da probóscide de Batracobdella gemmata. (b) Forma em divisão; (a) concentração de epimastigotas alongadas; (c) epimastigotas alongadas. Aumento de $1600 \mathrm{x}$.

Revta bras. Zool. 10 (3): 417-426, 1993 


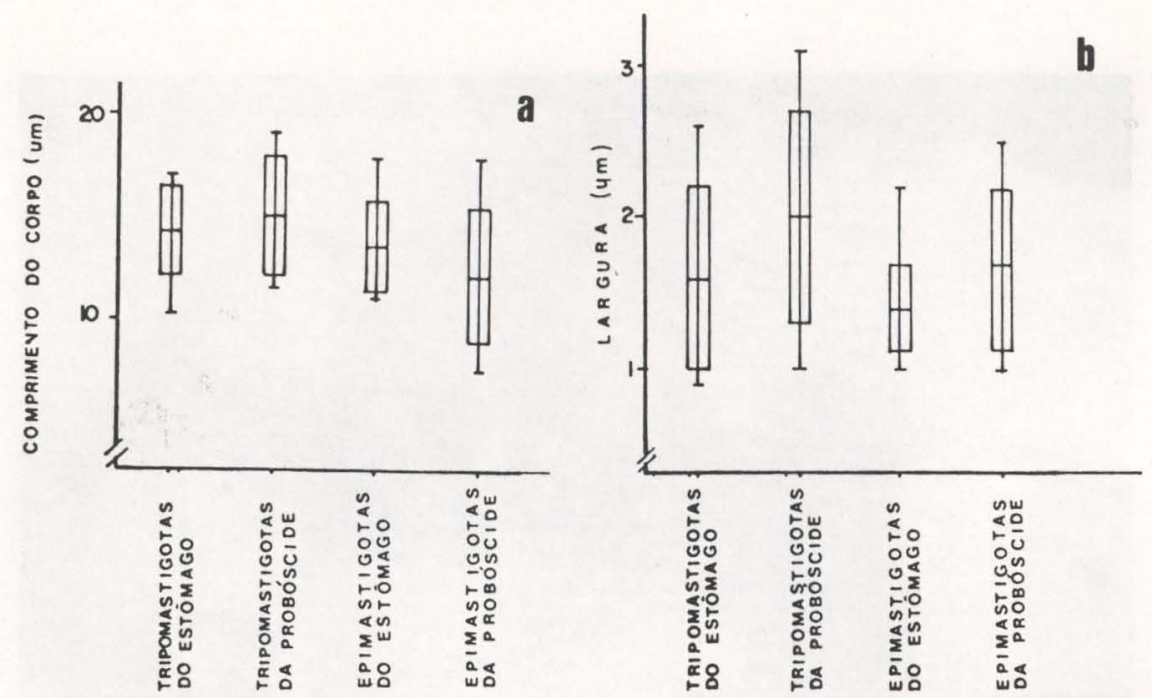

Fig. 6. Comparação gráfica entre o comprimento (a) e largura (b) do corpo de formas de tripanosomas do estômago e da probóscide de Batracobdella gemmata.

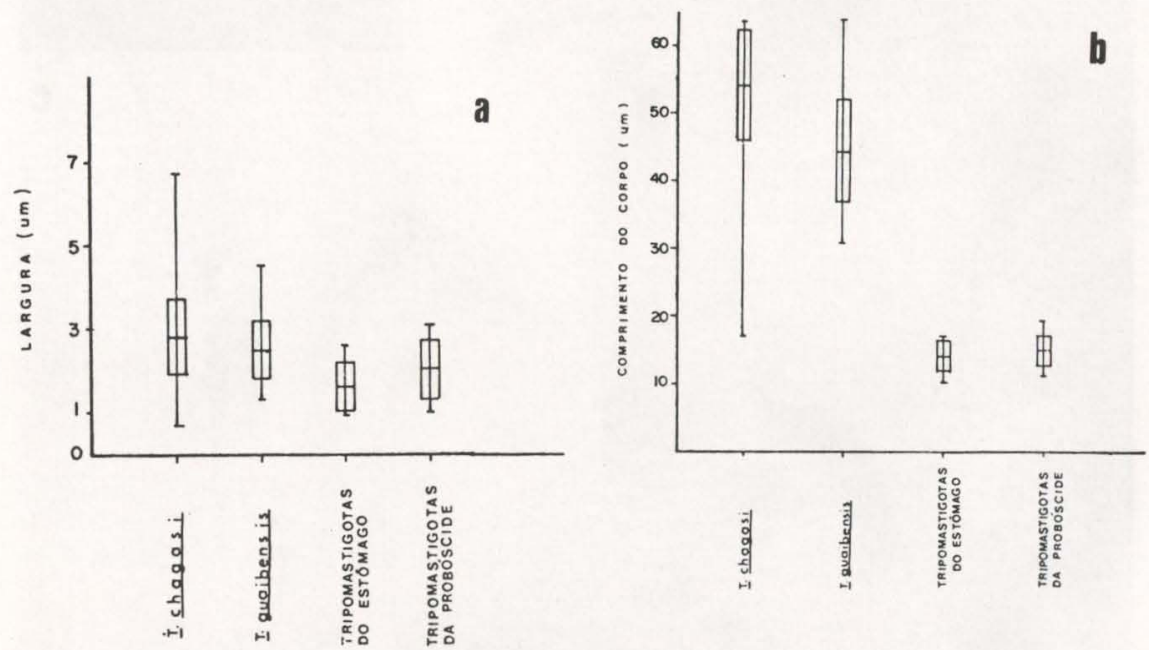

Fig. 7. Comparação gráfica entre a largura (a) e o comprimento (b) do corpo de Trypanosoma chagasi e Trypanosoma guaibensis e de tripomastigotas do estômago e da probóscide de Batracobdella gemmata.

cesso de divisão dos tripanosomas no estômago. As diferentes formas em divisão observadas nas sanguessugas capturadas no Lago-Açú, com diversas etapas de desenvolvimento (Fig. 1), são semelhantes às apresentadas por QUADRI (1962b) para Trypanosoma striati em meio de cultura. As formas amastigotas ora relatadas não foram observadas por QUADRI (1962a,b) e LETCH (1980), 
mas foram referidas por KHAN (1976) como presentes no desenvolvimento de T. murmanensis em sanguessuga marinha do gênero Johanssonia.

QUADRI (1962a,b) decreveu formas longas e finas semelhantes as agora encotradas (Fig. 5b,c) que poderiam ser etapas finais do desenvolvimento dos tripanosomas no estômago das sanguessugas e estar relacionadas com as formas longas observadas na probóscide. Essas formas na probóscide foram obtidas de sanguessugas mantidas em laboratório sem contato com hospedeiro, e tais observações coincidem com as de LETCH (1980) quanto ao aspecto alongado dos tripanosomas metacíclicos. As formas longas, por comparação aos resultados de QUADRI (1962a, b) e LETCH (1980) seriam as de maior maturidade e, provavelmente, as tripomastigotas representem as formas metacíclicas.

\section{REFERÊNCIAS BIBLIOGRÁFICAS}

ABOLARIN, M.O. 1970. A note on the trypanosomes from the African freshwater fish and some comments on the possible relationship between taxonomy and pathology in trypanosomes. Bull. Epizoot. Dis. Africa 18: 221-228.

BAKER, J.R. 1960. Trypanosomes and dactylosomes from the blood of freshwater fish east Africa. Parasitology 50: 515-526.

BARA, M.A. \& N.M. SERRA FREIRE. 1984. Infecção natural de sanguessugas do gênero Diplobdella por tripanosomas de Hypostomus punctatus Valencienne (Osteichthyes, Loricariidae). Rev. Fac. Vet. UFF 1: 55-57.

- 1985. Aspectos epidemiológicos de infecção por tripanosomas em Hypostomus punctatus no Lago-Açú da UFRRJ. Rev. Bras. Med. Vet. 7: 46-49.

BRUMPT, M.E. 1904. Contribution a l'etude de l'evolution des hemogrégarines et des trypanosomes. C. R. Séanc. Soc. Biol. 57: 165-167.

321-332.

1905. Trypanosomes et trypanoplasmes. Revue. Scient., Paris, 4:

1906a. Expériences relatives au mode de transmission des trypanosomes et des trypanoplasmes par les hirudinées. C. R. Séanc. Soc. Biol., Paris, 60: 77-79.

1906b. Sur quelques espécies nouvelles de trypanosomes parasites de poissons d'eau douce, leur mode d'evolution. C. R. Séanc. Soc. Biol., Paris, 60: $160-162$.

DIAS, E. \& L. FREITAS. 1943. Introdução ao estudo biométrico dos hemoflagelos do gênero Schizotrypanum. Mem. Inst. Oswaldo Cruz 38: 427-435.

DICE, L.R. \& H.J. LERAAS. 1936. A grafic method for comparing several sets of measurements. Contrib. Lab. Univ. Michigan 3: 1-3.

KHAN, R.A. 1976. The life cycle of Trypanosoma murmanensis Nikitin. Can J. Zool. 55: 1840-1849.

1977. Infectivity of Trypanosoma murmanensis to leeches (Johanssomia sp.). Can J. Zool. 55: 1968-1700.

LETCH, C.A. 1977. Leeches (Hemiclepsis marginata) as vector of fish trypano- 
somes. Tr. Roy. Soc. Trop. Med. Hyg. 71: 380-381.

1980. The life-cycle of Trypanosoma cobitis Mitrophanow, 1883. Parasitology 80: 163-170.

NEEDHAM, E.A. 1969. Trypanosoma tincae: some ecological aspects. Parasitology 59: $8 \mathrm{p}$.

QUADRI, S.S. 1962a. An experimental study of the life cycle of Trypanosoma danilewskyi in the leech, Hemiclepsis marginata. J. Protozool. 9: 254-258. . 1962b. The development in culture of Trypanosoma striati from a Indian fish. Parasitology 52: 229-235.

ROBERTSON, M. 1910. Further notes on a trypanosome found in the alimentary tract of Pontobdella muricata. Q. J. Microsc. Sci. 54: 119-139.

. 1912. Transmission of flagellates living in the blood of certain fresh water fishes. Phil. Trans. R. Soc. Lond. 202b: 29-50.

Recebido em 30.X. 1991; aceito em 25.XI.1993. 